\title{
Determinación de horarios de aberturas en una vivienda de construcción masiva para su corrección térmica interior
}

\section{Determination of opening schedules in massive construction housing for interior thermal correction}

\author{
DIAZ-SALAZAR, Arlees Ysrael†*, OJEDA-SANCHEZ, Jorge Armando y BOJÓRQUEZ- \\ MORALES, Gonzalo
}

ID $1^{\text {er }}$ Autor: Arlees Ysrael, Diaz-Salazar / ORC ID: 0000-003-4278-0597, Researcher ID Thomson: G-8094-2018, CVU CONACYT ID: 490740

ID $1^{\text {er }}$ Coautor: Jorge Armando, Ojeda-Sanchez / ORC ID: 0000-0002-5026-5459, Researcher ID Thomson: O-43812017, CVU CONACYT ID: 169799

ID $2^{\text {do }}$ Coautor: Gonzalo, Bojórquez-Morales / ORC ID: 0000-0001-9303-9278, Researcher ID Thomson: C-8687-2018 CVU CONACYT ID: 79645

DOI: $10.35429 / J A D .2019 .10 .3 .1 .10$

Recibido 21 de Octubre, 2019; Aceptado 15 de Diciembre, 2019

\begin{abstract}
Resumen
Actualmente la elección de modelos de construcción masiva es la alternativa con mayor recurrencia para adquirir una vivienda en clima cálido húmedo en México, sin embargo, gran parte de esta no se encuentra climáticamente adaptada y ocasiona incomodidad térmica en el usuario a causa del ambiente interior generado como respuesta de las características de su envolvente arquitectónica. Para corregir en breve tiempo el efecto de incomodidad térmica, el usuario generalmente recurre a soluciones de climatización mecanizada como ventiladores o aires acondicionados. Se propuso el empleo de horarios de aberturas en la envolvente arquitectónica en un clima cálido húmedo, principalmente en ventanas, como estrategia de corrección del ambiente térmico interior, por considerar sus ventajas como la aplicación inmediata y fácil implementación, en contraste con alguna modificación arquitectónica o física de la vivienda. Se efectuaron mediciones de temperatura de bulbo seco y humedad relativa en un modelo de vivienda de construcción masiva para determinar su comportamiento térmico en el mes más caluroso del año. Posteriormente se realizaron simulaciones con EnergyPlus para determinar horarios de abertura que fueran adecuados y corregir el ambiente térmico por prolongar las horas de confort interior al compararlos con el comportamiento termico interior original de la envolvente.
\end{abstract}

Desempeño térmico, Cálido húmedo, Simulación

\begin{abstract}
Currently the choice of massive construction models is the alternative with greater recurrence to acquire a house in warm humid climate in Mexico, however, much of this is not adapted to its climatic environment and causes thermal discomfort in the user because of the internal environment generated as response of the characteristics of its architectural envelope. To correct in a short time the effect of thermal discomfort, the user usually employs mechanized climatizations solutions such as fans or air conditioners. It was proposed the use of schedule for opening hours in the architectural envelope in a warm humid climate, mainly in windows, as a strategy to correct the internal thermal environment, considering its advantages as the immediate application and easy implementation, in contrast to some architectural or physical modification of the envelope. Dry bulb temperature and relative humidity measurements were made in a massive construction model to determine its thermal performance in the hottest month of the year. Subsequently, simulations were made with EnergyPlus to determine suitable opening times to correct the thermal environment by prolonging the hours of interior comfort when compared with the original interior thermal performance of the envelope.
\end{abstract}

Thermal performance, Warm humid climate, Simulation

Citación: DIAZ-SALAZAR, Arlees Ysrael, OJEDA-SANCHEZ, Jorge Armando y BOJÓRQUEZ-MORALES, Gonzalo. Determinación de horarios de aberturas en una vivienda de construcción masiva para su corrección térmica interior. Revista de Arquitectura y Diseño. 2019, 3-10: 1-10

\footnotetext{
*Correspondencia al Autor (Correo Electrónico: arleesdiaz@gmail.com)

$\dagger$ Investigador contribuyendo como primer autor.
} 


\section{Introducción}

El presente artículo se desarrolla en la temática de la arquitectura bioclimática y la adaptación al clima de la envolvente arquitectónica, y tiene por objetivo la determinación de mejoras de ambientes térmicos interiores mediante la implementación de la estrategia pasiva del horario de aberturas, principalmente ventanas, para beneficio del usuario de vivienda de construcción masiva.

La investigación aporta resultados y conocimiento al campo de la adecuación bioclimática de la envolvente arquitectónica, y la contribución es la determinación de un horario de aberturas adecuado que conlleva a una mejora en el ambiente térmico interior en el mes de mayor calor en clima cálido húmedo en Mérida, Yucatán, México.

Inicialmente se considera que un criterio para adaptar bioclimáticamente la envolvente arquitectónica, es el conocimiento de las características de los materiales ante el clima y el efecto en las condiciones de temperatura y humedad interiores que pueden ser generadas. Sin embargo, gran parte de la vivienda construída en serie, ya edificada, no se encuentra climáticamente adaptada y ocasiona incomodidad térmica en el usuario a causa del ambiente interior generado como respuesta de las características de su envolvente arquitectónica, en consecuencia, es necesario recurrir a correcciones de fácil implementación como medio para alcanzar resultados inmediatos.

Adicionalmente, el usuario no siempre puede recurrir a soluciones de climatización mecanizada como ventiladores 0 aires acondicionados para lograr rápidamente la mejora. Por lo tanto, la estrategia pasiva de recurrir a un horario de aberturas específico, se propone como una alternativa viable con resultados inmediatos.

En modelos de vivienda de construcción masiva en la ciudad de Mérida, Yucatán, durante la mayor parte del día predomina la falta de confort por calor en el interior de la vivienda, lo cual se debe a las condiciones de elevado calor y humedad de la envolvente arquitectónica durante las horas de sol, y liberación durante la noche, efecto de la masa térmica del sistema constructivo utilizado.
Por lo anterior, resulta necesario estudiar posibilidades de mejoramiento de las condiciones térmicas del ambiente interior de modelos de vivienda para determinar soluciones requeridas en el replanteamiento, corrección y optimización de dichos modelos y acercar o mantener sus condiciones térmicas interiores durante más tiempo en la zona de confort.

La afectación de los ambientes térmicos interiores en la envolvente construída a causa de su comportamiento térmico y materiales, puede ser corregida con el empleo de la estrategia de ventilación natural y por métodos pasivos. En envolventes de vivienda de construcción masiva, el mejoramiento de la condición interior puede lograrse mediante el control de la abertura de ventanas y permitir flujo de aire al interior.

Para el clima cálido húmedo y sobre todo durante su período más caluroso, la abertura indiscriminada podría no ser suficiente para satisfacer necesidades de confort térmico en los espacios interiores de la vivienda, por lo que emplear la estrategia de ventilación al interior mediante la selección y determinación de un horario de aberturas resulta una herramienta adecuada para mejoramiento de las condiciones térmicas interiores, y un indicador mediante el que se pueda discriminar el uso de sistemas mecánicos de climatización como elemento complementario.

El análisis del horario de aberturas de ventanas puede realizarse mediante el programa de computadora EnergyPlus, que con parámetros de ventilación dados, se determina la apertura de ventanas en un edificio como una estrategia de control mediante el modulo de administración de sistemas de energía, donde se puede incluir la ventilación natural, mecánica o mixta (Berkeley et al., 2017).

De tal manera que dicha herramienta de análisis energético para edificios, permite definir parámetros de diseño específicos, a partir de implementar programación del consumo y comportamiento de los usuarios de dicho edificio, en algunos casos mediante modelos estadísticos del consumo de los usuarios de dicho edificio (Yao, 2018). 
Como ejemplo Belleri et al., 2014, analizaron la ventilación natural de un edificio de oficinas a partir de un estudio de campo mediante el registro del uso de ventanas por los ocupantes, condiciones climáticas locales, condiciones interiores y cambios de aire. Su objetivo principal fue determinar el parámetro de diseño con mayor impacto en la incertidumbre asociada con el desempeño de la ventilación. Mediante simulaciones con la herramienta EnergyPlus y pruebas en túnel de viento, se verificó que el control de abertura de ventanas, junto con los coeficientes de presión ayudan a mejorar las predicciones del comportamiento de la ventilación natural (Belleri, Lollini, \& Dutton, 2014).

D'Oca y Hong, 2014, analizaron la relación entre las actividades de los usuarios y el consumo de energia en un edificio de oficinas, mediante análisis estadístico identificaron patrones de aberturas de ventanas. Descubrieron que los usuarios empleaban horarios de aberturas definidos de acuerdo a la falta de confort térmico interior y también por hábitos y rutina diaria, concluyeron que el impacto al abrir la ventana conlleva a la reduccion en el uso de energía, mejora del confort térmico y de la productividad.

Fabi et al., 2012, mediante revisión de literatura determinaron que el control de abertura y cerrado de ventanas resulta una actividad compleja que depende de factores en los que se vinculan las condiciones de su contexto exterior, las características y propiedades de la envolvente arquitectónica, y tiene un impacto en las condiciones internas que se desean alcanzar en edificios residenciales y de oficinas.

Jeong et al., 2016, identificaron en su estudio la relacion entre las actividades diarias de los ocupantes respecto al control de aberturas de ventanas, también verificaron la influencia por variables ambientales que relacionaron con el comportamiento de abrir y cerrar las ventanas. Encontraron que la abertura de ventanas esta relacionada con las actividades diarias de los usuarios en horas específicas y depende de las condiciones interiores, mientras que el cerrado se asoció con la disminución de la temperatura interior después de abrir las ventanas.
Lai et al., 2018, realizaron un estudio para diferentes edificios de departamentos en cinco diferentes zonas climáticas en China, consideraron el clima de las regiones, las temporadas, el tipo de edificio y los patrones de interaccion de los ocupantes en la abertura de ventanas. Observaron que los patrones de actividad en los horarios de aberturas de ventanas son diferentes durante los primeros cinco días de la semana respecto a los fines de semana cuya abertura se realiza mas tarde, y definieron un horario de aberturas enfocado para los dormitorios.

Less et al., 2019, emplearon simulación para comprobar el desempeño energético mediante ventilación basada en la temperatura exterior de casas ubicadas en California. El desempeño varió dependiendo de las características climáticas de los sitios estudiados, el modelo de vivienda y su hermeticidad. Tambien verificaron el ahorro energetico según rangos de ventilacion definidos por temporada climática y flujos de viento modulados dentro de cada temporada.

Sorgato et al., 2016, mediante simulación en EnergyPlus, concluyeron un horario de aberturas definido con empleo complementario de aire acondicionado para mejoramiento térmico interior en edificios residenciales en Brasil, y se consideró la masa térmica del edificio como influencia para un horario de aberturas adecuado para los ocupantes.

Wang \& Greenberg, 2015, demostraron a partir de simulaciones con EnergyPlus mediante un análisis con dos modos de ventilación, natural y modo mixto en un edificio de tamaño medio de oficinas en un clima cálido húmedo, que las estrategias de operación para ventanas en un horario especifico son una herramienta con un impacto directo en el confort y el consumo eficiente de energía de sistemas climatizados.

Zhao et al., 2014, evaluaron horarios de actividad para edificios de oficinas en diferentes zonas climáticas con simulación en EnergyPlus, consideraron que la ocupación del usuario tiene un impacto por la emisión de calor en el interior de los edificios, y que las condiciones interiores del edificio deben ser adecuadas para permanencia de la salud, productividad y confort. 
Concluyeron que los horarios de ocupación impactan en el uso del aire acondicionado y los consumos de energía, los cuales varían según zonas climáticas.

Zhao et al., 2016, utilizaron simulación en EnergyPlus para optimizar el consumo energético del aire acondicionado con base en la determinación de preferencias de confort térmico de los ocupantes. En la simulación implementaron un sistema de enfriamiento en modo mixto para ventilación $\mathrm{y}$ aire acondicionado, ajustado de acuerdo a un horario de ocupación y preferencias de confort térmico de los usuarios, y verificaron el impacto en el consumo energético del edificio.

\section{Condiciones climáticas}

La ciudad de Mérida, capital del estado de Yucatán, se ubica a $20^{\circ} 59^{\prime}$ latitud norte, $89^{\circ} 39^{\prime}$ de longitud oeste y altitud promedio de $11 \mathrm{msnm}$. El trabajo se realizó para el tipo climático propio de la ciudad Aw0 correspondiente al clima cálido subhúmedo, el de menor humedad, con lluvias en verano, se presentan cinco meses lluviosos que van de junio a octubre y siete meses secos (Instituto de Geografía, 1986:67). El registro de temperatura media normal anual es de $26.3^{\circ} \mathrm{C}$, y $3.9^{\circ} \mathrm{C}$ de diferencia entre la temperatura media normal de $24.3^{\circ} \mathrm{C}$ del mes más frío, enero, y de $28.2^{\circ} \mathrm{C}$ del mes más caliente, mayo, que también registró $36^{\circ} \mathrm{C}$ como temperatura máxima normal (SMN y CONAGUA, 2011).

\section{Características de la vivienda de construcción masiva estudiada}

La vivienda tiene un solo nivel y cuenta con sala-comedor, cocina, dos recámaras y un baño (figuras 1 y 2). La envolvente se encuentra constituida en sus muros por paneles de concreto armado de $0.10 \mathrm{~m}$ y el techo es $0.22 \mathrm{~m}$ de espesor conformado por vigueta y bovedilla hueca de concreto. La altura interior de piso a plafón terminado es de $2.40 \mathrm{~m}$.

La orientación de la vivienda respecto al Norte es de $28^{\circ}$ o $208^{\circ}$, con su eje largo ubicado al Noreste-Suroeste y su fachada principal está dirigida hacia el Noreste.

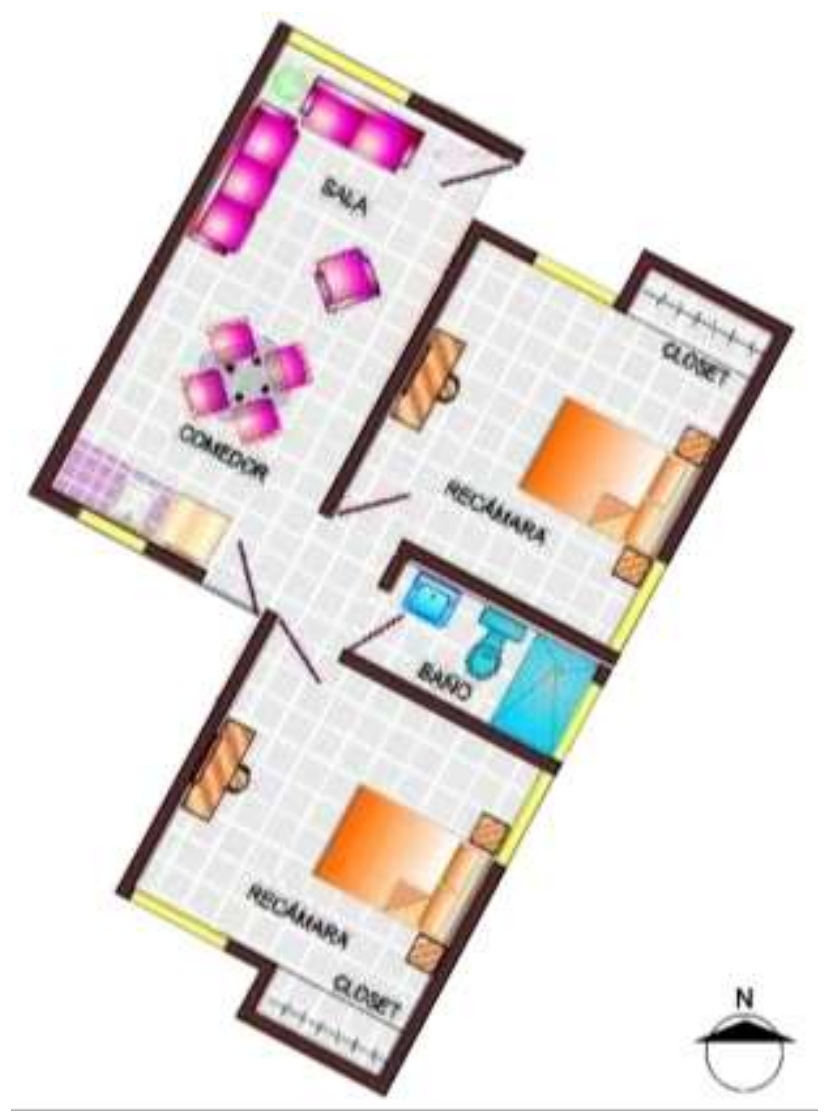

Figura 1 Planta arquitectónica del modelo de vivienda estudiado con la orientación que presenta en el fraccionamiento

Fuente: Arlees Díaz, archivo personal

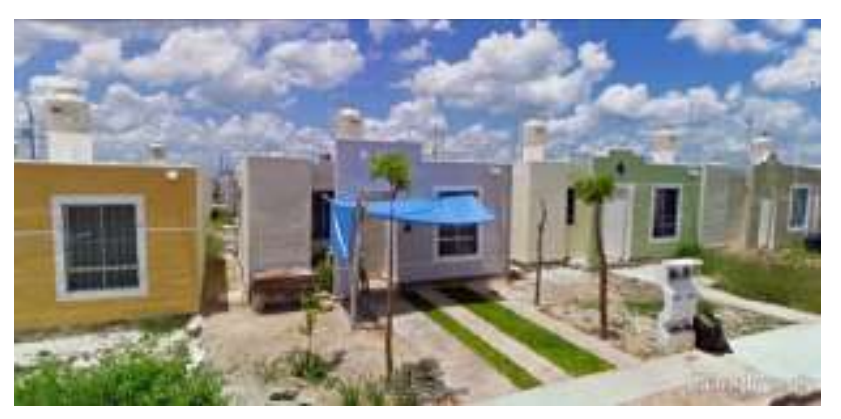

Figura 2 Modelo de vivienda estudiado, la vivienda del centro fue la monitoreada

Fuente: Imagen tomada de Google Earth, modificada por Arlees Díaz

\section{Metodología}

En la ciudad de Mérida, Yucatán, se eligió con base en su repetición, utilización de sistemas constructivos y dimensionamiento, un modelo de vivienda de construcción masiva unifamiliar de una planta y dos recámaras perteneciente a uno de los fraccionamientos más extensos y representativos. Por monitoreo y medición de datos se determinaron las condiciones termicas de temperatura de bulbo seco y humedad relativa interiores y exteriores de la vivienda durante el mes más caluroso, correspondiente al mes de mayo. 
Las condiciones térmicas se obtuvieron mediante el uso de datalogger U12-012 de OnsetComp, que presentan una exactitud en temperatura de $\pm 0.35^{\circ} \mathrm{C}$ en el rango típico de temperaturas de Mérida y de $\pm 2.5 \%$ en humedad relativa de entre $10 \%$ al $90 \%$.

Después se realizó el diagnóstico del comportamiento térmico de la envolvente y se verificó la afectación de su ambiente interior mediante la evaluación con un modelo de confort seleccionado.

Las condiciones térmicas al interior de la casa en estudio, así como las condiciones exteriores de temperatura exterior mencionadas anteriormente, pueden apreciarse en la Gráfica 1.

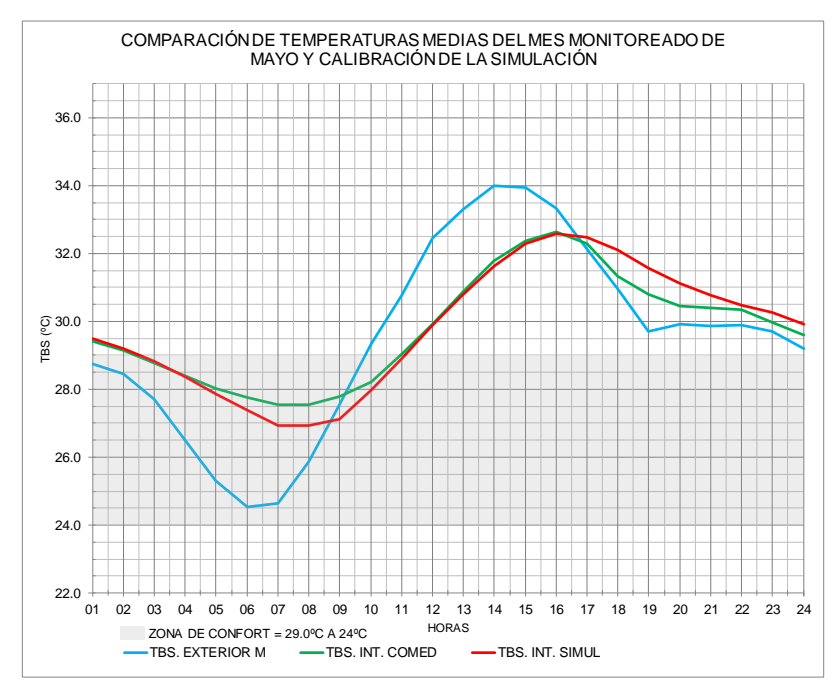

Gráfica 1 Medición de las condiciones térmicas interiores y exteriores de la casa habitación en estudio Fuente: Elaboración de los autores

La zona de confort empleada como parámetro de evaluación de los ambientes higrotérmicos interiores, se determinó con base en las ecuaciones del enfoque adaptativo de la ANSI/ASHRAE, 2013, para calcular la temperatura neutral "Tn", y el límite inferior y superior para el $90 \%$ de las personas, donde "Tma" es la temperatura media del aire del clima local:

$\mathrm{Tn}=0.31 * \mathrm{Tma}+17.8$

Límite inferior $=0.31 * \mathrm{Tma}+15.3$

Límite superior $=0.31 * \mathrm{Tma}+20.3$
Respecto a las temperaturas medias (Tm) fueron obtenidas de la hoja de Normales Climatológicas de la CONAGUA período 1981-2010 (SMN y CONAGUA, 2011), se tiene:

Para mayo, con $\mathrm{Tm}=28.2^{\circ} \mathrm{C}$, la temperatura neutral es $26.5^{\circ} \mathrm{C}$, los límites son $29.0^{\circ} \mathrm{C}$ a $24.0^{\circ} \mathrm{C}$. La zona de confort definida fue calculada para condiciones sedentarias, ropa ligera acorde a la que se usa en la región, y los límites de humedad relativa de $20 \%$ a $75 \%$.

Posteriormente, se empleó el programa de computadora EnergyPlus para la simulación térmica digital con base en las zonas $\mathrm{o}$ ambientes térmicos generados por los espacios de la vivienda, geometría, aberturas, materiales de su envolvente y propiedades térmicas de estos. El modelo digital de simulación, fue creado, configurado y validado respecto a los datos de temperatura de bulbo seco y humedad relativa medidos, que se tomaron como parámetro inicial de referencia para comparar con los resultados obtenidos por simulación, como establecen en su metodología de calibración y validación Monfet et. al., 2009. Adicionalmente se verificó la certeza del tiempo de retraso térmico de la simulación y concordó con los datos de retraso térmico reportados por Szokolay, 2004.

Se simularon las condiciones térmicas de la vivienda con sus materiales originales y las diferentes alternativas según los horarios de abertura propuestos para el posterior análisis del comportamiento térmico y la determinación de la opción más benéfica para el mes de mayor calor respecto al uso de la estrategia pasiva del horario de aberturas.

Para determinar los horarios de aberturas y su pertinencia, las 24 horas del día fueron separadas en tres turnos de ocho horas cada uno. Los turnos fueron establecidos por las actividades en los períodos activos y de descanso de los habitantes. Los horarios definidos se pueden apreciar en la Tabla 1.

\begin{tabular}{|l|l|}
\hline \multicolumn{1}{|c|}{ Actividad } & Horario \\
\hline Turno 1 - Mañana & 00:00 a 08:00 hrs. \\
\hline Turno 2 - Tarde & 08:00 a 16:00 hrs. \\
\hline Turno 3 - Noche & 16:00 a 24:00 hrs. \\
\hline
\end{tabular}

Tabla 1 Horarios de actividad y descanso de los habitantes de la casa habitación en estudio

Fuente: Elaboración de los autores 
El porcentaje de abertura en ventanas, considerado para la simulación numérica, correspondiente a un $0 \%$ para ventanas completamente cerradas, $50 \%$ para ventanas semiabiertas y de $100 \%$ para ventanas completamente abiertas. Los criterios y porcentajes de abertura corresponden a una simulación por cada horario.

\begin{tabular}{|l|l|l|l|}
\hline $\begin{array}{c}\text { No. de } \\
\text { simulación }\end{array}$ & $\begin{array}{c}\text { Horario 1 } \\
\text { 00:00- } \\
\text { 08:00hrs. } \\
\text { \% apertura }\end{array}$ & $\begin{array}{c}\text { Horario 2 } \\
\text { 00:80- } \\
\text { 16:00hrs. } \\
\text { \% apertura }\end{array}$ & $\begin{array}{c}\text { Horario 3 } \\
\text { 16:00- } \\
\text { 24:00hrs. } \\
\text { \% } \\
\text { apertura }\end{array}$ \\
\hline 1 & 50 & 0 & 0 \\
\hline 2 & 100 & 0 & 0 \\
\hline 3 & 0 & 50 & 0 \\
\hline 4 & 0 & 100 & 0 \\
\hline 5 & 0 & 0 & 50 \\
\hline 6 & 0 & 0 & 100 \\
\hline 7 & 50 & 50 & 0 \\
\hline 8 & 100 & 100 & 0 \\
\hline 9 & 0 & 50 & 50 \\
\hline 10 & 0 & 100 & 100 \\
\hline 11 & 50 & 0 & 50 \\
\hline 12 & 100 & 0 & 100 \\
\hline 13 & 0 & 0 & 0 \\
\hline 14 & 50 & 50 & 50 \\
\hline 15 & 100 & 100 & 100 \\
\hline
\end{tabular}

Tabla 2 Horarios de abertura propuestos y porcentaje de abertura en cada turno.

Fuente: Elaboración de los autores explicación:

La anterior tabla tiene la siguiente Ventanas abiertas en un turno.

1. 50-0-0 (abertura por la mañana)

2. 100-0-0 (abertura por la mañana)

3. 0-50-0 (abertura por la tarde)

4. 0-100-0 (abertura por la tarde)

5. $0-0-50$ (abertura por la noche)

6. $0-0-100$ (abertura por la noche)

Ventanas abiertas en dos turnos.

7. 50-50-0 (abertura por la mañana y tarde)

8. 100-100-0 (abertura por la mañana y tarde)

9. 0-50-50 (abertura por la tarde y noche)

10. $0-100-100$ (abertura por la tarde y noche)

11. 50-0-50 (abertura por la mañana y noche)

12. 100-0-100 (abertura por la mañana y noche)
Ventanas abiertas en tres turnos.

13. 0-0-0 (ventanas al $0 \%$ o cerradas en los tres turnos)

14. 50-50-50 (ventanas al 50\% en los tres turnos)

15. 100-100-100 (ventanas al 100\% o siempre abiertas en los tres turnos).

\section{Resultados}

Se presentan para mayo gráficas promedio mensuales de temperatura de bulbo seco y humedad relativa, de acuerdo a la implementación de ventanas abiertas en un turno con las simulaciones 1 a 6 (Gráficas 2 y $3)$, ventanas abiertas en dos turnos con las simulaciones 7 a 12 (Gráficas 4 y 5), y ventanas abiertas en tres turnos con las simulaciones 13, 14 y 15 (Gráficas 6 y 7). En la Figura 3, se muestran los mejores resultados.

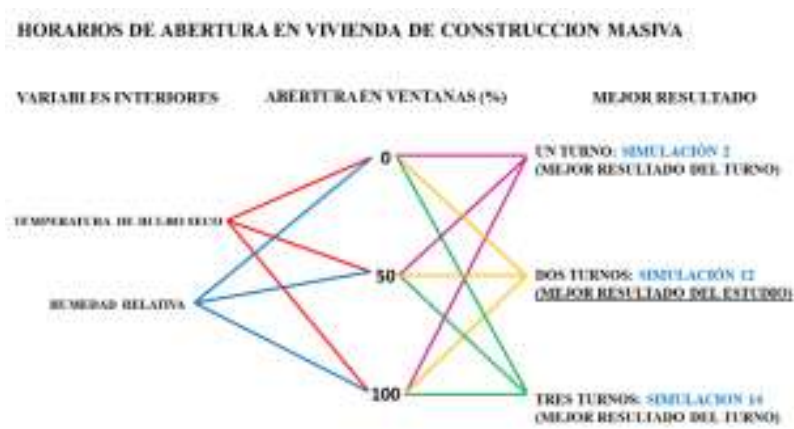

Figura 3 Mejores resultados del desempeño térmico interior de la vivienda en cada turno

Fuente: Elaboración de los autores

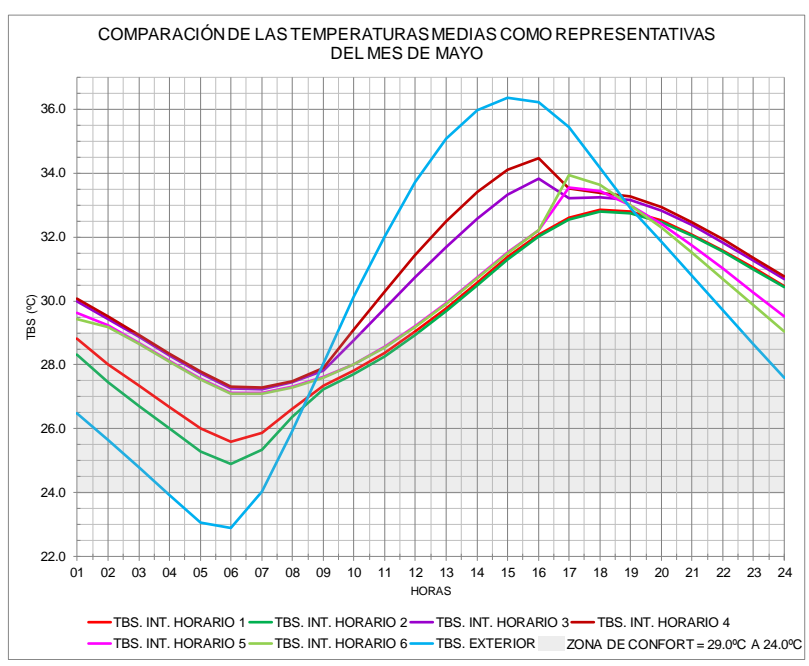

Gráfica 2 Variabilidad de las temperaturas de bulbo seco medias con la implementación de ventanas abiertas en un turno

Fuente: Elaboración de los autores 


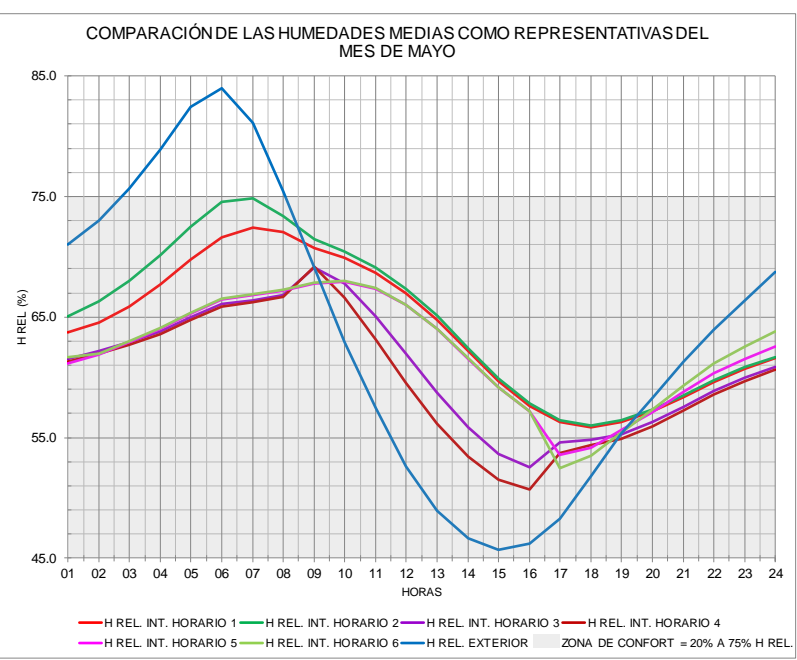

Gráfica 3 Variabilidad de las humedades relativas medias con la implementación de ventanas abiertas en un turno

Fuente: Elaboración de los autores

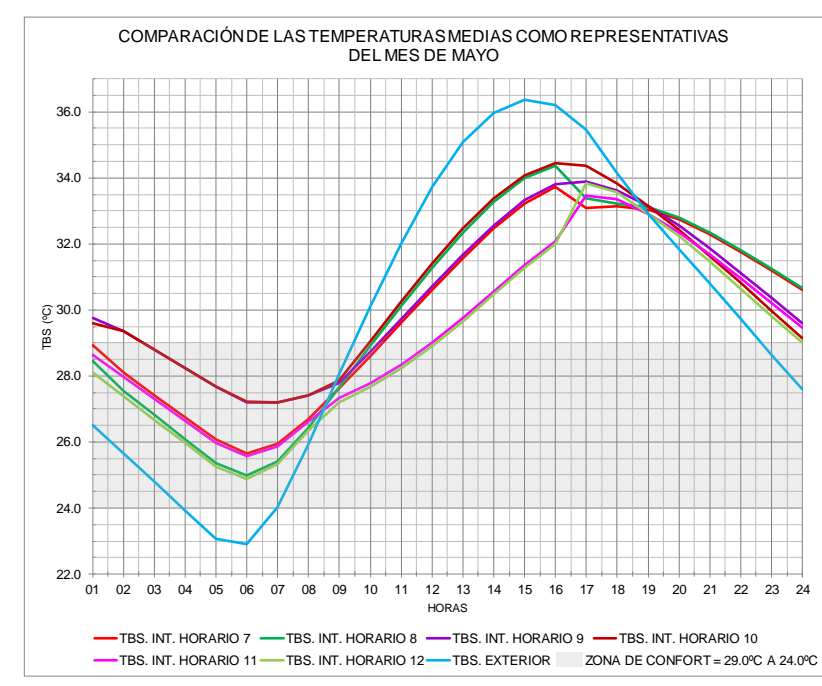

Gráfica 4 Variabilidad de las temperaturas de bulbo seco medias con la implementación de ventanas abiertas en dos turnos.

Fuente: Elaboración de los autores

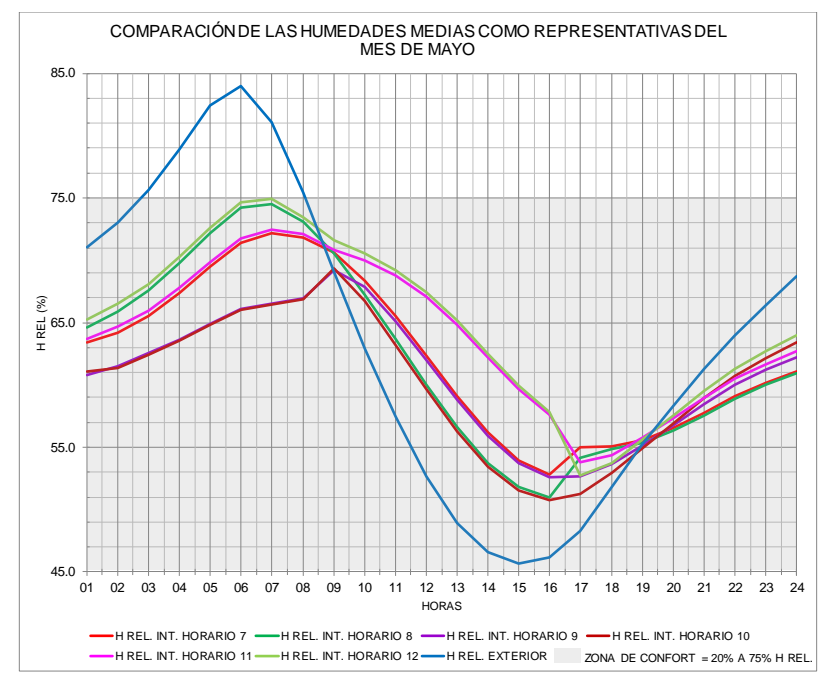

Gráfica 5 Variabilidad de las humedades relativas medias con la implementación de ventanas abiertas en dos turnos

Fuente: Elaboración de los autores

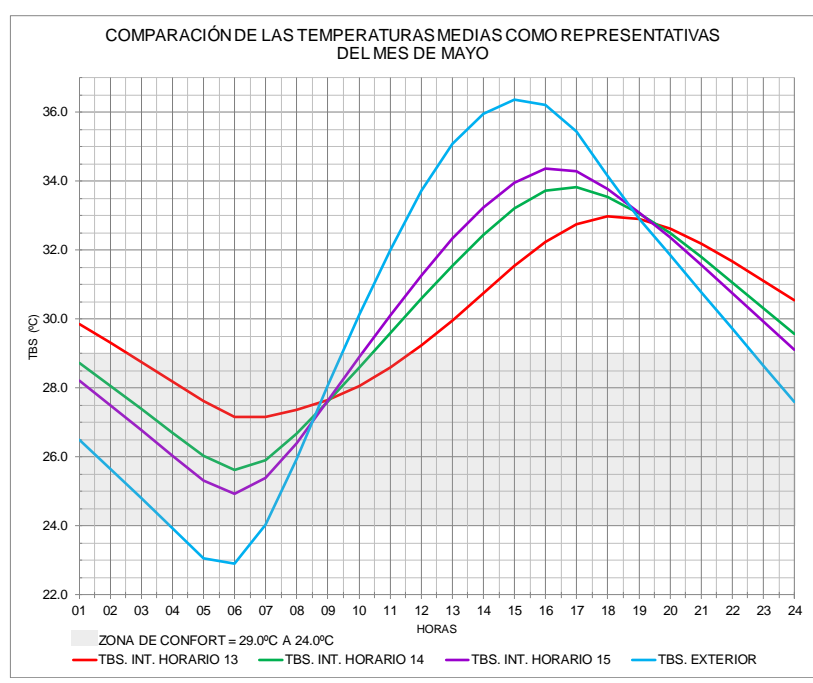

Gráfica 6 Variabilidad de las temperaturas de bulbo seco medias con la implementación de ventanas abiertas en tres turnos

Fuente: Elaboración de los autores

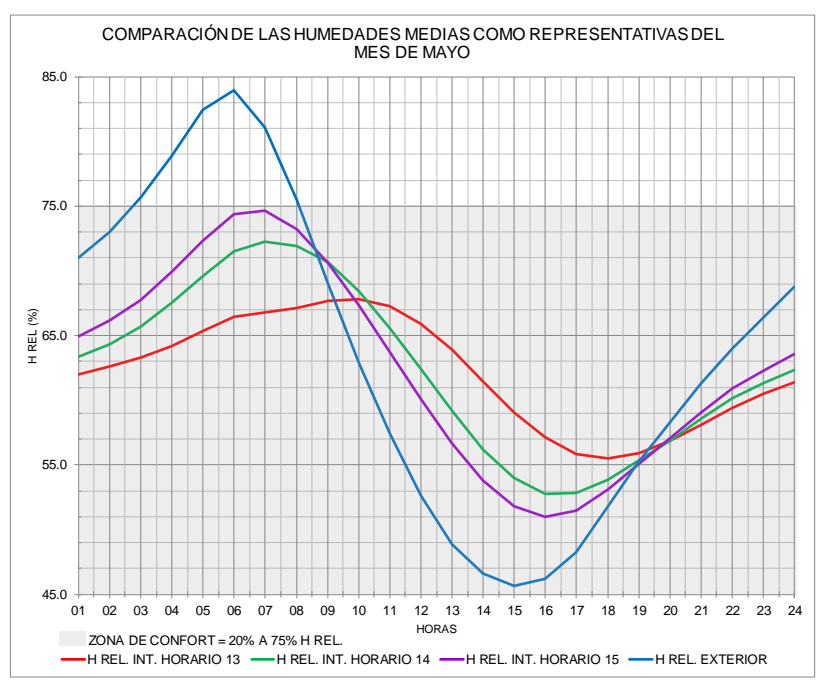

Gráfica 7 Variabilidad de las humedades relativas medias con la implementación de ventanas abiertas en tres turnos

Fuente: Elaboración de los autores

La implementación de aberturas durante un turno (Gráficas 2 y 3), demostró mejora, específicamente para la simulación 2 , que fue mantener completamente abiertas las ventanas en el horario de 00:00-08:00 h por mantener condiciones de confort durante 12 horas, de 00:00 a 12:00 h, y las temperaturas reducidas con la mínima temperatura a $24.9^{\circ} \mathrm{C}$ a las $6: 00$ h.

Seguidamente la simulación 1, demostró el mismo tiempo de 12 horas dentro de la zona de confort con temperaturas ligeramente más elevadas con la mínima de $25.6^{\circ} \mathrm{C}$ a las $6: 00 \mathrm{~h}$. $\mathrm{Se}$ considera que cerrar en los horarios posteriores mantiene la humedad dentro de la casa con la disminución de temperatura. 
Sin embargo, posterior a la hora 12:00 inicia la elevación de la temperatura fuera de la zona de confort establecida para el interior de la vivienda.

Para este mes, las condiciones son incómodas durante la mitad del día, de las 12:00 a las 24:00 h, e incluye períodos de descanso del habitante, la humedad superior al $50 \%$ incrementa la condición opresiva en el organismo por altas temperaturas. $\mathrm{La}$ incomodidad se eleva más de $3.5^{\circ} \mathrm{C}$ del límite superior de confort, y alcanza su máxima a las 18:00 h.

La implementación de aberturas durante dos turnos (Gráficas 4 y 5), demostró la mayor mejora del estudio. Específicamente para la simulación 12, que fue mantener completamente abiertas las ventanas en el horario de 00:00-08:00 h y abrirlas nuevamente en el horario de 16:00-24:00 $\mathrm{h}$ por mantener condiciones de confort durante 12.4 horas y las temperaturas reducidas, con la mínima temperatura a $24.9^{\circ} \mathrm{C}$ a las $6: 00 \mathrm{~h}$. Se considera propicio dejar abiertas las ventanas en los dos turnos de estos horarios, primero por el aire fresco que entra a primeras horas del día y posteriormente por la disipación de la temperatura almacenada por la envolvente a causa de las horas con sol mediante el intercambio convectivo de la noche.

Con respecto a los horarios de abertura implementados para los tres turnos (Gráficas 6 y 7), se identificó con igual duración de 10 horas en la zona de confort, a la simulación 13 (mantener cerradas en los tres turnos) y 15 (mantener al 100\% abiertas en los tres turnos), contrariamente a lo que se consideraría, durante el mes de mayo no se sugiere tener las ventanas abiertas por completo en los tres turnos (simulación 15) por la entrada del intenso calor exterior al ambiente interior de la envolvente. El mejor horario con duración de 10.5 horas en la zona de confort fue la simulación 14 por mantener las ventanas abiertas al $50 \%$ en los tres turnos.

Los resultados demuestran mejoramiento con la determinación de un horario de aberturas, además de certidumbre en la estrategia de ventilación y con una discriminación en el control al abrir ventanas y permitir el paso del aire.
Sin embargo, no resulta suficiente recurrir únicamente al horario de aberturas como estrategia de adaptación pasiva para mantener el confort por mas de 12.4 horas para el mes de mayo.

\section{Conclusiones}

Después de analizar las gráficas de temperatura y humedad de los diferentes períodos de estudio, se determinó que para la temporada cálida seca funciona mejor dos turnos de horarios de aberturas, específicamente mantener completamente abiertas las ventanas en el horario de 00:00-08:00 h y abrirlas nuevamente en el horario de 16:00-24:00 h, por lograr mejores condiciones higrotérmicas interiores al ubicarse más horas dentro de la zona de confort determinada durante el período de estudio.

Sin embargo, en las condiciones higrotérmicas evaluadas mediante el parámetro de confort de la ANSI/ASHRAE, 2013, para dicho período cálido seco y el mes más caluroso de todo el año, mayo, resultaron cómodas durante 12.4 horas solo con el uso del horario de aberturas por lo que es necesaria la implementación de estrategias adicionales para el mejoramiento de las condiciones térmicas interiores y prolongación en la zona de confort.

Para la temporada cálida seca los mejores resultados alcanzados fueron con condiciones interiores de comodidad por 12.4 horas. Por lo que la estrategia pasiva de horarios de abertura es térmicamente ineficiente al menos para el período mas caluroso del año, lo cual brinda una guía como complemento para el uso asistido por ventiladores antes que recurir a climatización por aire acondicionado.

Por último, la condición de incomodidad queda evidenciada con la gran necesidad interna de ventilación, para mitigar las condiciones de calor interno y la ineficiente ventilación natural que se tiene a causa del característico confinamiento de la envolvente de las viviendas de construcción masiva.

Resulta favorable la verificación, la especificación, y el refinamiento de horarios de aberturas en meses diferentes al mes crítico de mayo. 
La estrategia de determinación de un horario de aberturas conlleva a certidumbre en el control de ventanas y resulta útil de implementar en casos donde la envolvente arquitectónica sea difícilmente modificable por economía de recursos o por estructura física edilicia. Es una estrategia en la que apegados a un control conlleva a un beneficio inmediato pero limitado.

\section{Recomendaciones}

Apegarse a un horario de aberturas queda sujeto al período o temporada climática de estudio por lo que, para el clima cálido húmedo, con el objetivo de determinar una mejora, pueden ser diferentes para el mes de mayor fresco y el mes de mayor calor.

Adicionalmente, resulta recomendable la exploración de nuevos horarios de abertura en consideración de las necesidades y actividades del usuario, orientación del modelo de construcción masiva, distribución y tamaño de las ventanas, $y$ por las condiciones ambientales exteriores de la vivienda.

Se requiere más investigación para el refinamiento de horarios de aberturas, así como la exploración de horarios más reducidos o prolongados los cuales dependerán de las actividades de los usuarios.

Los horarios deben ser con mayor detalle, discriminando el cerrado de las ventanas según su orientación y considerar las horas en que las viviendas reciben soleado directo en las fachadas con ventanas para ajustar la entrada de ganancia térmica al interior de la envolvente.

Complementariamente al mes más caluroso y seco, mayo, se recomienda considerar los horarios de aberturas para los meses de la temporada climática de fresco o menor calor, y de la temporada de calor húmedo.

\section{Referencias}

ANSI/ASHRAE. (2013). ANSI/ASHRAE Standard 55-2013 Thermal Environmental Conditions for Human Occupancy. Atlanta: American Society of Heating, Refrigerating and Air-Conditioning Engineers, Inc.
Belleri, A., Lollini, R., \& Dutton, S. M. (2014). Natural ventilation design: An analysis of predicted and measured performance. Building and Environment, 81, 123-138, Elsevier. http://doi.org/10.1016/j.buildenv.2014.06.009

Berkeley, L., Ridge, O. A. K., Ut-battelle, M. B. Y., For, A., Energy, S., Or, D., ... The, W. (2017). Engineering Reference-Energy Plus.

D'Oca, s., \& Hong, T., (2014). A data-mining approach to discover patterns of window opening and closing behavior in offices. Building and Environment, 82, 726-739, Elsevier.

http://doi.org/10.1016/j.buildenv.2014.10.021

Fabi, V., Andersen, R., Corgnati, S., \& Olesen, B. (2012). Occupants' window opening behaviour: A literature review of factors influencing occupant behaviour and models. Building and Environment, 58, 188-198, Elsevier.

http://doi.org/10.1016/j.buildenv.2012.07.009

Instituto de Geografía. (1986). Ciudades alternativas para la descentralización. México: UNAM, UAM, DGIA.

Jeong, B., Jeong, J., \& Park, J. (2016). Occupant behavior regarding the manual control of windows in residential buildings. Energy and Buildings, 127, 206-216, Elsevier. http://doi.org/10.1016/j.enbuild.2016.05.097

Lai, D., Jia, S., Qi, Y., \& Liu, J. (2018). Window-opening behavior in Chinese residential buildings across different climate zones. Building and Environment, 142, 234243 ,

Elsevier. http://doi.org/10.1016/j.buildenv.2018.06.030

Less, B., Dutton, S., Walker, I., Sherman, M., \& Clark, J. (2019). Energy savings with outdoor temperature-based smart ventilation control strategies in advanced California homes. Energy and Buildings, 194, 317-327, Elsevier.

http://doi.org/10.1016/j.enbuild.2019.04.028

Monfet, D., Charneux, R., Zmeureanu, R., Lemire, N. (2009). Calibration of a building energy model using measured data. ASHRAE Transactions, 115, pt.1, 348-359. Chicago 2009. 
SMN y CONAGUA. (2011). Normales climatológicas para Mérida, Yucatán, período 1981-2010. Servicio Meteorológico Nacional y Comisión Nacional del Agua. http://smn.cna.gob.mx/tools/RESOURCES/Nor males8110/NORMAL31019.TXT

Sorgato, M., A.P. Melo, A., \& Lamberts, R. (2016). The effect of window opening ventilation control on residential building energy consumption. Energy and Buildings,133, 1-13, Elsevier. http://doi.org/10.1016/j.enbuild.2016.09.059

Szokolay, S. (2004). Introduction to Architectural Science the basis of sustainable design. Elsevier Science. Architectural Press. Burlington, Great Britain.

Wang, L., \& Greenberg, S. (2015). Window operation and impacts on building energy consumption. Energy and Buildings, 92, 313321 ,

Elsevier. http://doi.org/10.1016/j.enbuild.2015.01.060

Yao, J. (2018). Modelling and simulating occupant behaviour on air conditioning in residential buildings. Energy and Buildings, 175, 1-10, Elsevier. http://doi.org/10.1016/j.enbuild.2018.07.013

Zhao, J., Lasternas, B., Lam, K., Yun, R., \& Loftness, V. (2014). Occupant behavior and schedule modeling for building energy simulation through office appliance power consumption data mining. Energy and Buildings, 82, 341-355, Elsevier. http://doi.org/10.1016/j.enbuild.2014.07.033

Zhao, J., Lam, K., Ydstie, B., \& Loftness, V. (2016). Occupant-oriented mixed-mode EnergyPlus predictive control simulation. Energy and Buildings, 117, 362-371, Elsevier. http://doi.org/10.1016/j.enbuild.2015.09.027 